\title{
Anestesia por infusão contínua de propofol associado ao remifentanil em gatos pré-tratados com acepromazina
}

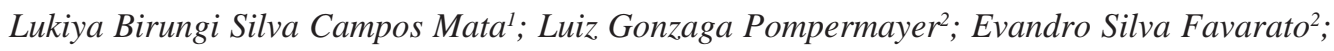 \\ Jair Duarte da Costa Junior ${ }^{3}$; Cinthya Dessaune Neves ${ }^{4}$;aciana Pereira ${ }^{4}$ e Napoleão Martins Argolo Neto ${ }^{2}$
}

\section{RESUMO}

O presente trabalho avaliou a associação anestésica do propofol, um anestésico geral de ação ultracurta e metabolismo rápido, ao remifentanil, opioide de grande poder analgésico, que potencializa os anestésicos gerais. O objetivo principal foi desenvolver um protocolo anestésico de uso intravenoso, por infusão contínua, que proporcione conforto ao paciente, segurança, com grande relaxamento muscular e analgesia em gatos submetidos a cirurgias eletivas como a ovariosalpingoisterectomia e orquiectomia. Foram utilizados 30 gatos, aleatoriamente distribuídos em 3 grupos de 10 animais. Em todos eles realizou-se a tranquilização com acepromazina (0,1 mg/kg, IM) e indução anestésica com propofol (6 mg/kg, IV). No grupo 1, a anestesia foi mantida por infusão contínua de propofol (0,6 mg/kg/min), no grupo 2, com propofol (0,4 mg/kg/min) e remifentanil ( $0,1 \mu \mathrm{g} / \mathrm{kg} / \mathrm{min})$, e no grupo 3, com propofol (0,4 mg/kg/min) e remifentanil $(0,2 \mu \mathrm{g} / \mathrm{kg} / \mathrm{min})$. As variáveis mensuradas foram: temperatura corporal, frequência cardíaca, frequência respiratória, saturação da oxiemoglobina, pressão arterial sistólica, hemogasometria arterial, analgesia e miorrelaxamento. Após análise dos resultados, pode-se concluir que o remifentanil promoveu incremento da analgesia, permitindo redução de $33 \%$ na velocidade de infusão do propofol, entretanto a infusão de $0,2 \mu \mathrm{g} / \mathrm{kg} / \mathrm{min}$ de remifentanil determinou maior depressão respiratória quando comparada à infusão de $0,1 \mu \mathrm{g} / \mathrm{kg} / \mathrm{min}$.

Palavras-chave: Anestesia, hemogasometria, analgesia, propofol, remifentanil, felinos.

\section{ABSTRACT}

\section{Anesthesia by continuous infusion of propofol with remifentanil in cats pretreated with acepromazine}

The present work evaluated the anesthetic association of propofol, an ultra-short acting general anesthetic with a fast metabolism, with remifentanil, an opioid of great analgesic potency that is used as adjunct to general anesthetics. The main objective was to develop a safe protocol of intravenous continuous infusion capable of providing comfort for the patient with great analgesic action and muscle relaxation for cats subjected to elective surgeries like ovariohysterectomy and orchiectomy. Thirty cats were randomly distributed in 3 groups of 10 animals each. All animals were tranquilized with acepromazine $(0.1 \mathrm{mg} / \mathrm{kg} \mathrm{IM})$ and general anesthesia was induced using propofol (6mg $/ \mathrm{kg}, \mathrm{IV})$. In group 1, general anesthesia was maintained by continuous infusion of propofol ( $0.6 \mathrm{mg} / \mathrm{kg} / \mathrm{min})$; in group 2, with propofol ( $0.4 \mathrm{mg} / \mathrm{kg} / \mathrm{min})$ and remifentanil $(0.1 \mu \mathrm{g} / \mathrm{kg} / \mathrm{min})$ and; in group 3, with propofol ( $0.4 \mathrm{mg} / \mathrm{kg} / \mathrm{min})$ and remifentanil

\footnotetext{
Recebido para publicação em dezembro de 2007 e aprovado em soutubro de 2010

Medica Veterinária, Doutora. Universidade Federal de Viçosa, Campus Universitário, Av. PH Rolfs, s/n, Centro, 36.570-000, Viçosa, Minas Gerais, Brasil. lukiyacampos@hotmail.com,

${ }^{2}$ Médico Veterinário, Doutor. Universidade Federal de Viçosa, Campus Universitário. Avenida PH Rolfs, s/n, Centro, 36.570-000, Viçosa, Minas Gerais, Brasil. lgonzaga@ufv.br, evandrofavarato@hotmail.com, argolo_napoleao@hotmail.com

${ }_{3}^{3}$ Médico Veterinário, Mestre. Universidade Federal de Viçosa, Campus Universitário. Av. PH Rolfs, s/n, Centro, 36.570-000, Viçosa, Minas Gerais, Brasil jairdjr@hotmail.com ${ }^{4}$ Médica Veterinária, Mestre. Universidade Federal de Viçosa, Campus Universitário. Avenida PH Rolfs, s/n, Centro, 36.570-000, Viçosa, Minas Gerais, Brasil. cinthyaneves@hotmail.com, tacianapereira@hotmail.com
} 
$(0.2 \mu \mathrm{g} / \mathrm{kg} / \mathrm{min})$. The measured variables were: temperature, heart rate, respiratory rate, oxyhemoglobin saturation, systolic arterial pressure, arterial blood gas analysis, analgesia and myorelaxation. Results of the analyses led to the conclusion that remifentanil promoted an increase in analgesia, allowing a 33\% reduction on the rate of propofol infusion, although the infusion of $0.2 \mu \mathrm{g} / \mathrm{kg} / \mathrm{min}$ of remifentanil determined a greater respiratory depression when compared to the $0.1 \mu \mathrm{g} / \mathrm{kg} / \mathrm{min}$ infusion.

Key words: Anesthesia, hemosametry, analgesia, propofol, remifentanil, feline.

\section{INTRODUÇÃO}

O propofol é um anestésico geral derivado do alquilfenol, de ação ultracurta e metabolismo rápido (Duke, 1995). Em gatos, é utilizado para indução ou como agente de manutenção anestésica, em doses repetidas ou por infusão contínua, associado ou não a analgésicos (Souza et al., 2002; Souza et al., 2003).

O remifentanil é um opioide sintético de curta ação, derivado das fenilpiperidinas como o fentanil, alfentanil e sufentanil. Como estes, é um opioide $\mu$-agonista seletivo com efeito analgésico dose-dependente e sua potência é 20 a 30 vezes maior que a do alfentanil quando se avalia a perda da consciência e analgesia (Jhaveri et al., 1997; Glass et al., 1999).

O remifentanil apresenta farmacocinética bem singular, com metabolização por esterases inespecíficas presentes no sangue e nos tecidos, devido a um ramo lateral metiléster em sua cadeia química, tornando sua depuração dependente não apenas da função hepática (Burkle et al., 1996; Glass et al., 1999). Por apresentar período de ação curto, sem efeito cumulativo, é considerado o opioide mais apropriado para o uso em infusão contínua (Nora \& Fortis, 2001; Videira \& Cruz, 2004).

Em humanos, a associação do remifentanil ao propofol é considerada vantajosa, devido aos seus efeitos complementares (Hogue et al., 1996; Mertens et al., 2003; Videira \& Cruz, 2004). Em medicina veterinária, entretanto, essa associação é pouco difundida, sendo poucos os estudos dessa associação anestésica na espécie felina (Correa et al., 2007).

O presente trabalho teve como objetivo avaliar a anestesia produzida pela infusão contínua de propofol associado ao remifentanil em gatos submetidos à ovariosalpingoisterectomia e orquiectomia.

\section{MATERIAL E MÉTODOS}

Foram utilizados 30 gatos, com idade média de um ano e pesando 3,5 \pm 0,49 kg, provenientes da rotina cirúrgica do Hospital Veterinário da Universidade Federal de Viçosa, com indicação de orquiectomia e ovariosalpingois- terectomia, como cirurgia eletiva. Os animais foram distribuídos aleatoriamente em três grupos de 10, contendo número igual de machos e fêmeas.

Exame físico, hemograma completo e perfil bioquímico foram realizados em todos os animais no período pré-operatório, visando comprovar o bom estado de saúde. Somente participaram desse estudo animais hígidos e classificados como ASA I, segundo a American Society of Anesthesiologists, após aquiescência formal dos proprietários.

Após jejum hídrico de seis e alimentar de 12 horas, todos os animais receberam acepromazina ${ }^{5}$ por via intramuscular, na dose de $0,1 \mathrm{mg} / \mathrm{kg}$, como medicação préanestésica (MPA). Quinze minutos após a MPA, foi realizada a cateterização da veia cefálica e indução anestésica com $6 \mathrm{mg} / \mathrm{kg}$ de propofol ${ }^{6}$, por via intravenosa, e intubação orotraqueal. No grupo 1 (G1) a anestesia foi mantida por infusão contínua de propofol na velocidade de $0,6 \mathrm{mg} / \mathrm{kg} /$ min, no grupo 2 (G2) com propofol na velocidade de 0,4 $\mathrm{mg} / \mathrm{kg} / \mathrm{min}$, associado ao remifentanil ${ }^{7}$ na velocidade de $0,1 \mu \mathrm{g} / \mathrm{kg} / \mathrm{min}$, e no grupo 3 (G3) com propofol na velocidade de $0,4 \mathrm{mg} / \mathrm{kg} / \mathrm{min}$, associado ao remifentanil na velocidade de $0,2 \mu \mathrm{g} / \mathrm{kg} / \mathrm{min}$.

Para todos os grupos foram utilizadas bombas de infusão ${ }^{8}$, e nos grupos 2 e 3 foram usadas infusões independentes para o propofol e remifentanil. Iniciada a infusão contínua, os animais foram posicionados em decúbito dorsal e preparados para a cirurgia. O procedimento cirúrgico foi realizado sempre pela mesma equipe, padronizando-se assim o tempo de execução.

Foram registradas as seguintes variáveis: temperatura corporal com termômetro digital posicionado na mucosa retal; frequência cardíaca (FC) e saturação da oxiemoglobina $\left(\mathrm{SpO}_{2}\right)$, com oxímetro de pulso ${ }^{9}$, cujo sensor foi posicionado inicialmente na base da cauda e na língua após a indução da anestesia; pressão arte-

\footnotetext{
${ }^{5}$ Acepram 0,2\% - Univet S/A Indústria Veterinária

${ }^{6}$ Diprivan $1 \%$ - AstraZeneca

${ }^{7}$ Ultiva $1 \mathrm{mg}$ - Glaxo Smithkline

${ }^{8}$ Bomba de infusão LF 2001e FARS 600 - Lifemed Pesquisas Médicas Ind. E Com. Ltda.

${ }^{9}$ NPB 290 - Nellcor Puritan Bennett Europe BU
} 
rial sistólica (PAS), pelo método não invasivo com auxílio de um Doppler vascular ${ }^{10}$; frequência respiratória (FR), pela observação dos movimentos da parede do tórax; tempo de preenchimento capilar (TPC), pela pressão digital sobre a mucosa gengival; e hemogasometria arterial ${ }^{11}$, realizada em duas amostras colhidas da artéria uterina e testicular na fêmea e no macho, respectivamente aos 20 e 40 minutos após a indução (A1 e A2).

A analgesia foi classificada em suficiente e insuficiente e avaliada em duas etapas. A primeira realizada por meio de pinçamento cutâneo e a segunda por intermédio do procedimento cirúrgico, o qual foi indicado somente quando a analgesia foi considerada suficiente na primeira etapa de avaliação. Como parâmetros de avaliação foram registrados os valores de frequência cardíaca, a pressão arterial sistólica e frequência respiratória. A analgesia foi considerada insuficiente quando se observou uma elevação de aproximadamente $20 \%$ nos valores de um dos parâmetros.

O miorrelaxamento foi avaliado de acordo com o grau de relaxamento da musculatura abdominal e dos membros, classificado como ausente, moderado ou intenso. Também se verificou a presença de reflexos palpebral, corneal, interdigital e laringotraqueal.

O período de recuperação anestésica foi considerado o intervalo de tempo entre o término da infusão contínua e o início da deambulação.

Os momentos estabelecidos para mensuração das variáveis foram M0, antes da administração da medicação pré-anestésica (MPA); M1, 15 minutos após a MPA; M2, imediatamente após a indução anestésica e intubação orotraqueal; M3, 15 minutos após M2, após incisão da parede abdominal nas fêmeas ou do escroto nos machos; M4 30 minutos após M2, no momento da ligadura do primeiro pedículo ovariano nas fêmeas ou do primeiro cordão espermático nos machos; e M5 60 minutos após M2, ao término da cirurgia.

A análise estatística foi realizada para comparação entre as amostras (A1 e A2) nas variáveis hemogasométricas e para o período de recuperação anestésica, utilizando-se o teste t de Student. Para os demais dados paramétricos foi utilizada Análise de Variância (ANOVA), seguida pelo teste de Tukey.

Foram comparadas as diferenças entre os grupos em cada momento e as diferenças ao longo dos momentos em cada grupo individualmente.

Para todas as análises, considerou-se diferença estatística quando $\mathrm{p}<0,05$.

\footnotetext{
${ }^{10}$ Doppler Vascular Portátil - Medmega Indústria e Equipamentos Médicos LTDA ${ }^{11}$ Gasômetro ABL 5 - Radiometer / CopenHagen
}

\section{RESULTADOS E DISCUSSÃO}

Todos os animais estavam habituados ao convívio com pessoas e permitiram sem muita dificuldade o manuseio e a coleta de dados no M0. A tranquilização observada com a administração IM de acepromazina na dose de $0,1 \mathrm{mg} / \mathrm{kg}$ foi satisfatória em todos os animais, facilitando ainda mais a mensuração dos parâmetros em M1.

A indução foi rápida e tranquila em todos os animais com a dose de 6,0 mg/kg de propofol recomendada por Morgan \& Legge (1989) e Souza et al. (2003). Conforme relatado por Souza et al. (2003), não ficou evidenciada no presente experimento a apneia durante e após a indução, o que é considerado um inconveniente do propofol em gatos (Brearley et al., 1988; Morgan \& Legge, 1989; Andress et al., 1995; Sano et al., 2003). Durante a indução, da mesma forma que o observado por Souza et al. (2002) e Souza et al. (2003), foram evidenciadas manifestações de dor à injeção ou contrações musculares.

A velocidade de infusão do propofol de $0,6 \mathrm{mg} / \mathrm{kg} /$ min utilizada no G1 foi obtida em experimento piloto e considerada como a menor velocidade a promover plano anestésico adequado ao início do procedimento cirúrgico e analgesia cutânea. Entretanto, não coincide com as observações de Souza et al. (2003), que relatam a velocidade de $0,4 \mathrm{mg} / \mathrm{kg} / \mathrm{min}$ como sendo mínima para a produção de analgesia cutânea no gato, bem como da citação de Andress et al. (1995) que consideraram 0,5 $\mathrm{mg} / \mathrm{kg} / \mathrm{min}$ como suficiente para manutenção dos gatos intubados.

Durante a manutenção anestésica, em 50\% dos animais do G1 a analgesia foi considerada insuficiente nos momentos de maior estimulação dolorosa, necessitando de complementação da dose do propofol com "bolus" de $3 \mathrm{mg} / \mathrm{kg}$. Short \& Bufalari (1999) relatam que o propofol promove analgesia moderada, devendo ser associado a anestésicos locais, agentes anti-inflamatórios não esteroidais ou opioides, com a finalidade de potencializar a analgesia.

Nos grupos 2 e 3, a analgesia foi considerada suficiente em todos os momentos após a indução, mesmo com o propofol na velocidade de $0,4 \mathrm{mg} / \mathrm{kg} / \mathrm{min}$ demonstrando os efeitos analgésicos do remifentanil, característico dos opióides $\mu$-agonistas (Glass et al., 1999). Em outro estudo, Correa et al. (2007) também observaram maior analgesia com a associação do remifentanil $(0,3 \mu \mathrm{g} / \mathrm{kg} / \mathrm{min}$.) ao propofol (0,3 $\mathrm{mg} / \mathrm{kg} / \mathrm{min}$.) em cirurgias de ovariosalpingoisterectomia em gatas.

A velocidade de infusão do remifentanil de $0,1 \mu \mathrm{g} / \mathrm{kg} /$ min usada no G2 e de $0,2 \mu \mathrm{g} / \mathrm{kg} / \mathrm{min}$ no G3 promoveu aparentemente o mesmo grau de analgesia, não sendo observado efeito analgésico dose-dependente relatado por Jhaveri et al. (1997) e Glass et al. (1999). 
Os reflexos palpebral e corneal estiveram presentes em um número maior de animais do G2 e G3 do que do G1, o que pode ser atribuído ao menor grau de hipnose em decorrência da menor velocidade de infusão do propofol. Em humanos, é citado que o remifentanil possui efeito hipnótico satisfatório somente em doses a partir de $12 \mu \mathrm{g} /$ kg (Jhaveri et al., 1997).

A temperatura corporal declinou de forma significativa após a indução anestésica (Tabela 1 e Figura 1a) nos três grupos experimentais, o que pode ser atribuído à associação dos efeitos da acepromazina e do propofol, como observado por Souza et al. (2003). É relatado que o propofol deprime os centros medulares no SNC, incluindo o termorregulador (Fantoni et al., 1996). A redução da temperatura corporal também é frequente em animais medicados com acepromazina, devido à depleção de catecolaminas no hipotálamo, onde se processa o controle central da termorregulação (Booth \& Mcdonald, 1992), e pela vasodilatação periférica promovida por esse fármaco (Cortopassi \& Fantoni, 2002). Além disso, o relaxamento muscular considerado intenso para todos os animais, a incapacidade de produção de calor por tremores e redução do metabolismo devem ter contribuído para a queda da temperatura corporal (Yasbek, 2002). Não se observou interferência do remifentanil na temperatura corporal, visto que não houve diferença significativa entre grupos.

Após a indução anestésica, observou-se redução da frequência cardíaca nos três grupos (Tabela 1 e Figura 1b), demonstrando claramente a ação cronotrópica negativa do propofol citada por muitos autores (Brearley et al., 1988; Weaver \& Raptopoulos, 1990; Duke, 1995; Souza et al., 2002; Souza et al., 2003). Durante a manutenção anestésica, apenas no G1 observou-se redução significativa da frequência cardíaca, o que pode ser atribuído à maior velocidade de administração de propofol utilizada $(0,6 \mathrm{mg} / \mathrm{kg} /$ min). Não foi observada alteração na frequência cardíaca com a associação do remifentanil ao propofol nas velocidades de 0,1 e $0,2 \mu \mathrm{g} / \mathrm{kg} / \mathrm{min}$ nos grupos 2 e 3 , respectivamente. James et al. (1992) e Michelsen et al. (1996) relataram que o remifentanil promove redução da frequência cardíaca dose-dependente, e em velocidade acima de 0,6 $\mu \mathrm{g} / \mathrm{kg} / \mathrm{min}$ essa redução torna-se mais intensa.

Foi observada redução significativa da PAS no G2 e G3 após a indução anestésica (Tabela 1 e Figura 1c). A redução da PAS é citada por James et al. (1992) na anestesia com remifentanil e descrita por Murrel et al. (2005) com a associação propofol e remifentanil em cães. A redução da pressão arterial sistólica é promovida também por outros opioides $\mu$-agonistas e está relacionada à redução do tônus simpático e ao aumento do tônus vagal (James et al., 1992). Segundo Michelsen et al. (1996), quando se trata do remifentanil, esse achado tem pouca rele- vância clínica, pois devido ao seu rápido metabolismo, esses valores retornam rapidamente ao normal após o término da infusão, mesmo após 8 h de infusão.

Houve redução da FR em todos os grupos imediatamente após a indução anestésica (M2), sem diferença significativa entre eles (Tabela 1 e Figura 1d). A depressão respiratória é citada por outros autores na anestesia com propofol em gatos (Brearley et al., 1988; Souza et al., 2002; Souza et al., 2003), sendo atribuída ao efeito depressor do propofol sobre o centro respiratório (Langley \& Heel, 1988; Morgan \& Ledge, 1989; Fantoni et al., 1996; Souza et al.; 2003).

A saturação de oxiemoglobina declinou após a indução anestésica (M2) no G1 e G3 (Tabela 1 e Figura 1e), contudo, no G3 essa variável alcançou níveis abaixo dos considerados fisiológicos para a espécie em 30\% dos animais, que necessitaram de suplementação com oxigênio puro durante todos os momentos de avaliação (M2 a M5), podendo indicar que a velocidade de infusão do remifentanil tenha sido excessiva nesse grupo, pois segundo Nora \& Fortis (2001) o remifentanil promove depressão respiratória relacionada à dose e/ou a velocidade de administração. No G2 a saturação da oxiemoglobina também se manteve abaixo dos limites fisiológicos, contudo sem significado estatístico. Diante desses resultados, torna-se prudente a indicação de suplementação com oxigênio puro ou a instituição de respiração controlada quando o remifentanil fizer parte do protocolo anestésico (Murrel et al.,2005).

Na avaliação do pH(a) não foram encontradas diferenças significativas na comparação entre grupos (Tabela 2). Fato semelhante ocorreu nos grupos ao longo do tempo (A1 e A2). As discretas variações aconteceram dentro dos limites fisiológicos para gatos, que, segundo Middleton et al. (1981), varia de 7,21 a 7,44.

$\mathrm{A} \mathrm{pO}_{2}(\mathrm{a})$ também não apresentou diferenças significativas na comparação entre os grupos, nem entre as amostras (Tabela 2). Entretanto, nos animais do grupo 3 observaram-se valores elevados da $\mathrm{pO}_{2}$ (a) na A1 (105,49 mmHg). Esse achado possivelmente foi ocasionado pela suplementação de oxigênio aos animais nesse período. Nos animais do grupo 1, na A2 foi observado o valor médio de 80,76 mmHg, que, segundo McDonell (1996), é considerado hipoxemia. A provável causa para esse evento foi a hipoventilação promovida pelo propofol em razão da maior velocidade de infusão $(0,6 \mathrm{mg} / \mathrm{kg} / \mathrm{min})$ utilizada nesse grupo, semelhante aos relatos de Brearley et al. (1988), Souza et al. (2002) e Souza et al. (2003). Os demais valores encontraram-se dentro da faixa de referência que varia de 87,5 a 118 mmHg (Middleton et al., 1981).

Todas as médias observadas para a $\mathrm{pCO}_{2}$ (a) encontraram-se dentro dos limites fisiológicos para a espécie (Tabela 2), compreendido entre 26,5 a 41 mmHg (Middleton 
Tabela 1. Valores médios e desvios-padrão ( \pm ) da temperatura corporal (TC), freqüência cardíaca (FC), pressão arterial sistólica (PAS), freqüência respiratória (FR), saturação da oxiemoglobina (SpO2), volume corrente (Vt) e volume minuto (Vm) apresentados por gatos pré-tratados com acepromazina $(0,1 \mathrm{mg} / \mathrm{kg})$ e submetidos à indução anestésica com propofol (6,0 mg/kg) e manutenção com propofol 0,6 mg/kg/min (G1); propofol 0,4 mg/kg/min e remifentanil 0,1 $\mathrm{gg} / \mathrm{kg} / \mathrm{min}$ (G2); e propofol 0,4 mg/kg/min e remifentanil 0,2 $\mu \mathrm{g} / \mathrm{kg} / \mathrm{min}$ (G3) nos diferentes momentos de avaliação

\begin{tabular}{|c|c|c|c|c|c|c|c|}
\hline Parâmetros & Grupos & M0 & M1 & M2 & M3 & M4 & M5 \\
\hline \multirow[t]{3}{*}{$\mathrm{TC}\left({ }^{\circ} \mathrm{C}\right)$} & G1 & $38,93(0,43)^{\mathrm{A}, \mathrm{a}}$ & $38,7(0,4)^{\mathrm{A}, \mathrm{a}}$ & $38,46(0,4)^{\mathrm{A}, \mathrm{a}}$ & $37,24(1,1)^{\mathrm{A}, \mathrm{b}}$ & $36,49(1,0)^{\mathrm{A}, \mathrm{bc}}$ & $35,83(1,3)^{\mathrm{A}, \mathrm{c}}$ \\
\hline & G2 & $39,28(0,27)^{\mathrm{A}, \mathrm{a}}$ & $38,93(0,4)^{\mathrm{A}, \mathrm{ab}}$ & $38,19(0,8)^{\mathrm{A}, \mathrm{bc}}$ & $37,44(0,8)^{\mathrm{A}, \mathrm{cd}}$ & $36,78(0,8)^{\mathrm{A}, \mathrm{de}}$ & $36,08(0,8)^{A, e}$ \\
\hline & G3 & $39,05(0,49)^{\mathrm{A}, \mathrm{a}}$ & $38,9(0,4)^{\mathrm{A}, \mathrm{a}}$ & $38,66(0,4)^{\mathrm{A}, \mathrm{a}}$ & $37,38(0,6)^{\mathrm{A}, \mathrm{b}}$ & $36,84(0,6)^{\mathrm{A}, \mathrm{bc}}$ & $36,47(0,6)^{\mathrm{A}, \mathrm{c}}$ \\
\hline \multirow[t]{3}{*}{ FC(bat/min) } & G1 & $190,2(38,4)^{\mathrm{A}, \mathrm{ab}}$ & $214,8(20,3)^{\mathrm{A}, \mathrm{a}}$ & $171,9(22,3)^{\mathrm{A}, \mathrm{b}}$ & $160,8(22,8)^{\mathrm{A}, \mathrm{b}}$ & $177,8(29)^{\mathrm{A}, \mathrm{ab}}$ & $175,4(35,7)^{\mathrm{A}, \mathrm{b}}$ \\
\hline & G2 & $195,1(23,9)^{\mathrm{A}, \mathrm{a}}$ & $204,4(29,5)^{\mathrm{A}, \mathrm{a}}$ & $173(22,5)^{\mathrm{A}, \mathrm{a}}$ & $166,6(30,8)^{\mathrm{A}, \mathrm{a}}$ & $179(37,7)^{\mathrm{A}, \mathrm{a}}$ & $191,8(28,5)^{\mathrm{A}, \mathrm{a}}$ \\
\hline & G3 & $198(36,62)^{\mathrm{A}, \mathrm{a}}$ & $203(33,5)^{\mathrm{A}, \mathrm{a}}$ & $175(23,5)^{\mathrm{A}, \mathrm{a}}$ & $167,4(39,8)^{\mathrm{A}, \mathrm{a}}$ & $199,1(26,1)^{\mathrm{A}, \mathrm{a}}$ & $194,9(34,2)^{\mathrm{A}, \mathrm{a}}$ \\
\hline \multirow[t]{3}{*}{ PAS(mmHg) } & G1 & $139(24,2)^{\mathrm{A}, \mathrm{a}}$ & $126,1(33,7)^{\mathrm{A}, \mathrm{a}}$ & $102(37,9)^{\mathrm{A}, \mathrm{a}}$ & $91(26,1)^{\mathrm{A}, \mathrm{a}}$ & $123,3(48,9)^{\mathrm{A}, \mathrm{a}}$ & $115,8(44,2)^{\mathrm{A}, \mathrm{a}}$ \\
\hline & G2 & $143,2(35,3)^{\mathrm{A}, \mathrm{a}}$ & $116(38,1)^{\mathrm{A}, \mathrm{a}}$ & $95(21,7)^{\mathrm{A}, \mathrm{b}}$ & $88(37,4)^{\mathrm{A}, \mathrm{b}}$ & $102,8(29,8)^{\mathrm{A}, \mathrm{a}}$ & $123,8(39,8)^{\mathrm{A}, \mathrm{a}}$ \\
\hline & G3 & $155,5(28,9)^{\mathrm{A}, \mathrm{a}}$ & $118,5(18,8)^{\mathrm{A}, \mathrm{a}}$ & $100,5(34,8)^{\mathrm{A}, \mathrm{b}}$ & $111(37,1)^{\mathrm{A}, \mathrm{b}}$ & $132,5(35,4)^{\mathrm{A}, \mathrm{a}}$ & $125(28,5)^{\mathrm{A}, \mathrm{a}}$ \\
\hline \multirow[t]{3}{*}{ FR(mov/min) } & G1 & $62,7(28,3)^{\mathrm{A}, \mathrm{a}}$ & $43(14,32)^{\mathrm{A}, \mathrm{ab}}$ & $32,6(10,8)^{\mathrm{A}, \mathrm{bc}}$ & $27,8(9,5)^{\mathrm{A}, \mathrm{bc}}$ & $24,1(10,7)^{\mathrm{A}, \mathrm{bc}}$ & $20,9(8,5)^{\mathrm{A}, \mathrm{c}}$ \\
\hline & G2 & $57,8(13,3)^{\mathrm{A}, \mathrm{a}}$ & $50,4(26,3)^{\mathrm{A}, \mathrm{a}}$ & $29,2(8,3)^{\mathrm{A}, \mathrm{b}}$ & $22,5(6,4)^{\mathrm{A}, \mathrm{b}}$ & $19,9(6,3)^{\mathrm{A}, \mathrm{b}}$ & $20,1(6,9)^{\mathrm{A}, \mathrm{b}}$ \\
\hline & G3 & $73,3(25,7)^{\mathrm{A}, \mathrm{a}}$ & $61,7(15)^{\mathrm{A}, \mathrm{a}}$ & $24(7,9)^{\mathrm{A}, \mathrm{b}}$ & $21,1(7,5)^{\mathrm{A}, \mathrm{b}}$ & $20,8(6,2)^{\mathrm{A}, \mathrm{b}}$ & $20,4(5,5)^{\mathrm{A}, \mathrm{b}}$ \\
\hline \multirow[t]{3}{*}{$\mathrm{SpO}_{2}(\%)$} & G1 & $94,1(2,8)^{\mathrm{A}, \mathrm{a}}$ & $93,1(3)^{\mathrm{A}, \mathrm{ab}}$ & $88,8(6,9)^{\mathrm{A}, \mathrm{b}}$ & $91,6(2,4)^{\mathrm{A}, \mathrm{ab}}$ & $91(1,9)^{\mathrm{A}, \mathrm{ab}}$ & $90,9(2,9)^{\mathrm{A}, \mathrm{ab}}$ \\
\hline & G2 & $95,6(2,3)^{\mathrm{A}, \mathrm{a}}$ & $94,2(2,8)^{\mathrm{A}, \mathrm{a}}$ & $90,9(4,8)^{\mathrm{A}, \mathrm{a}}$ & $91,6(2,1)^{\mathrm{A}, \mathrm{a}}$ & $93,4(4,7)^{\mathrm{A}, \mathrm{a}}$ & $93,3(3,9)^{\mathrm{A}, \mathrm{a}}$ \\
\hline & G3 & $95,3(2,5)^{\mathrm{A}, \mathrm{a}}$ & $94,4(2,7)^{\mathrm{A}, \mathrm{a}}$ & $89,1(4,2)^{\mathrm{A}, \mathrm{b}}$ & $92,7(3,5)^{\mathrm{A}, \mathrm{ab}}$ & $93,7(3,9)^{\mathrm{A}, \mathrm{a}}$ & $93,2(2,9)^{\mathrm{A}, \mathrm{ab}}$ \\
\hline
\end{tabular}

Para cada variável, médias seguidas pela mesma letra minúscula na linha ou maiúscula na coluna, não diferem entre si pelo teste de Tukey $(\mathrm{p}<0,05)$. As letras minúsculas representam igualdade entre os momentos e as maiúsculas entre os grupos.
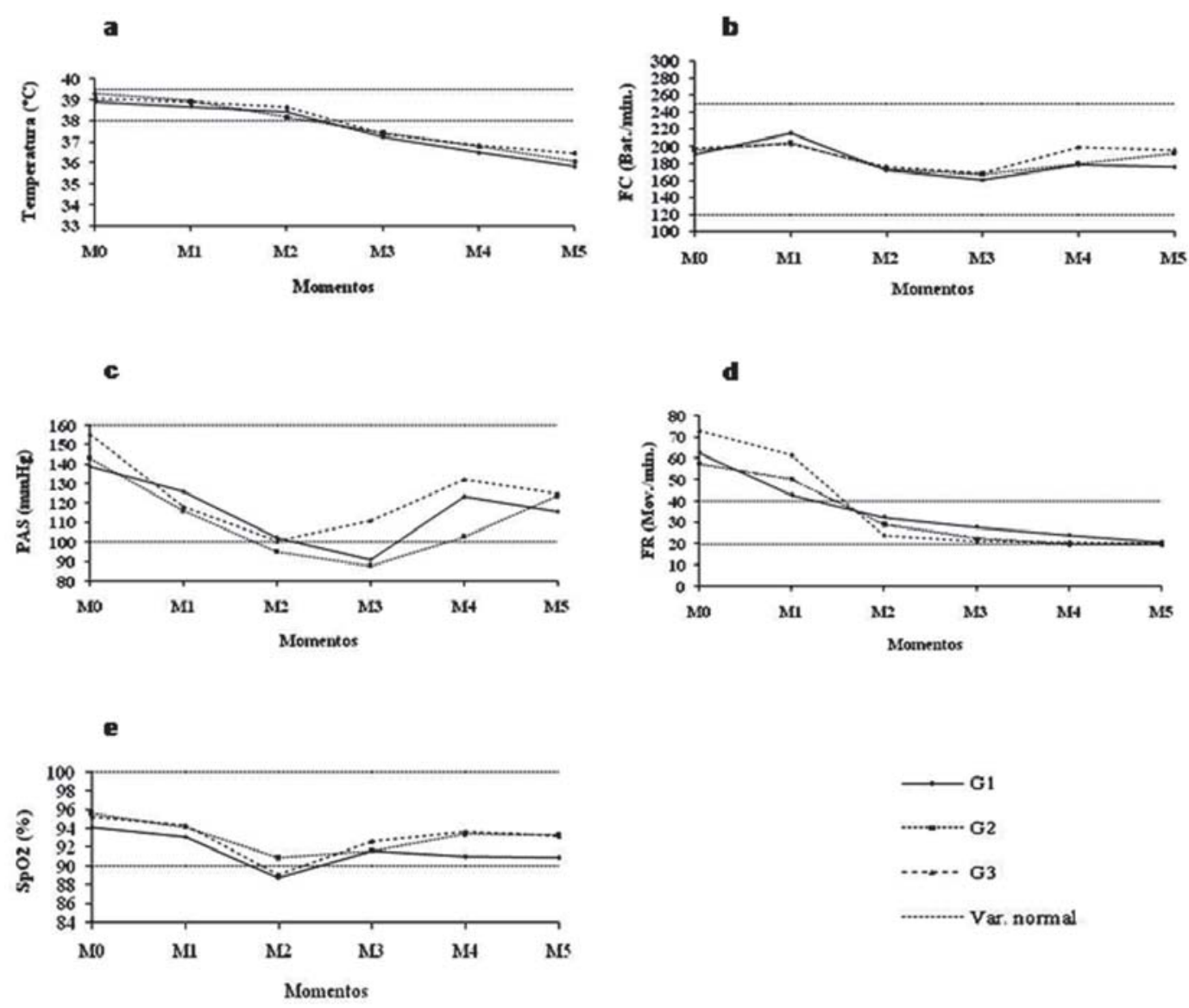

Figura 1. Representação dos valores médios da temperatura corporal (a), frequência cardíaca (b), pressão arterial sistólica (c), freqüência respiratória (d) e saturação de oxihemoglobina (e) apresentados por gatos pré-tratados com acepromazina $(0,1 \mathrm{mg} / \mathrm{kg})$ e submetidos à indução anestésica com propofol (6,0 mg/kg) e manutenção com propofol 0,6 mg/kg/min (G1); propofol 0,4 mg/kg/min e remifentanil 0,1 $\mu \mathrm{g} / \mathrm{kg} / \mathrm{min}$ (G2); e propofol 0,4 mg/kg/min e remifentanil 0,2 $\mu \mathrm{g} / \mathrm{kg} / \mathrm{min}$ (G3). 
et al., 1981). Nenhuma diferença estatística foi encontrada na comparação entre grupos ou mesmo entre os tempos dentro de um mesmo grupo.

O comportamento do $\mathrm{CHCO}_{3}(\mathrm{a}), \mathrm{ctCO}_{2} \mathrm{~T}(\mathrm{a})$ e da cBase(a) foram semelhantes (Tabela 2) e permaneceram dentro da faixa de normalidade para a espécie (Middleton et al., 1981).

A recuperação anestésica foi suave e livre de complicações em todos os animais. Observou-se menor tempo de recuperação dos grupos 2 (60,1 $\pm 34,4$ minutos) e 3 (53,3 \pm 18,97 minutos) em comparação com o grupo 1
(89,5 \pm 32,91 minutos). Tal constatação confirma o curto período de ação do remifentanil em decorrência da metabolização por esterases plasmáticas, resultando em rápida recuperação, mesmo após infusões prolongadas, como exposto por Glass et al. (1999). A maior dose de propofol utilizada no G1 pode ter sido a causa do prolongado período de recuperação apresentado por este grupo, pois em felinos a meia-vida de eliminação do propofol é aumentada quando esse é utilizado em altas doses ou por tempo prolongado (Hall \& Clark, 1991; Andress et al., 1995).

Tabela 2. Valores médios e desvios-padrão $( \pm)$ do $\mathrm{pH}$, da pressão parcial de oxigênio $\mathrm{pO}_{2}$ (a), da pressão parcial de dióxido de carbono ( $\left.\mathrm{pCO}_{2}\right)$, da concentração de bicarbonato plasmático $\left(\mathrm{cHCO}_{3}\right)$, da concentração total de dióxido de carbono ( $\left.\mathrm{ctCO}_{2} \mathrm{~T}\right)$ e da concentração de base (cBase) no sangue arterial de gatos pré-tratados com acepromazina $(0,1 \mathrm{mg} / \mathrm{kg})$ e submetidos à indução anestésica com propofol (6,0 mg/kg) e manutenção com propofol 0,6 mg/kg/min (G1), propofol 0,4 mg/kg/min e remifentanil 0,1 $\mu \mathrm{g} / \mathrm{kg} / \mathrm{min}$ (G2) e propofol 0,4 mg/kg/min e remifentanil 0,2 $\mu \mathrm{g} / \mathrm{kg} / \mathrm{min}$ (G3) nos diferentes momentos de avaliação

\begin{tabular}{|c|c|c|c|}
\hline Parâmetros & Grupos & A1 & A2 \\
\hline \multirow{3}{*}{$\mathrm{pH}$} & G1 & $7,35(0,048)^{\mathrm{A}, \mathrm{a}}$ & $7,37(0,071)^{\mathrm{A}, \mathrm{a}}$ \\
\hline & G2 & $7,31(0,044)^{\mathrm{A}, \mathrm{a}}$ & $7,32(0,050)^{\mathrm{A}, \mathrm{a}}$ \\
\hline & G3 & $7,34(0,066)^{\mathrm{A}, \mathrm{a}}$ & $7,33(0,048)^{\mathrm{A}, \mathrm{a}}$ \\
\hline \multirow{3}{*}{$\mathrm{pO}_{2}(\mathrm{mmHg})$} & G1 & $105,49(52,88)^{\mathrm{A}, \mathrm{a}}$ & $80,76(21,91)^{\mathrm{A}, \mathrm{a}}$ \\
\hline & G2 & $87,80(17,28)^{\mathrm{A}, \mathrm{a}}$ & $96,25(9,12)^{\mathrm{A}, \mathrm{a}}$ \\
\hline & G3 & $137,70(83,95)^{\mathrm{A}, \mathrm{a}}$ & $90,70(10,25)^{\mathrm{A}, \mathrm{a}}$ \\
\hline \multirow{3}{*}{$\mathrm{pCO}_{2}(\mathrm{mmHg})$} & G1 & $34,97(5,26)^{\mathrm{A}, \mathrm{a}}$ & $32,54(4,62)^{\mathrm{A}, \mathrm{a}}$ \\
\hline & G2 & $32,5(9,43)^{\mathrm{A}, \mathrm{a}}$ & $41(15,86)^{\mathrm{A}, \mathrm{a}}$ \\
\hline & G3 & $37,2(7,85)^{\mathrm{A}, \mathrm{a}}$ & $38,3(5,64)^{A, ~ a ~}$ \\
\hline \multirow{3}{*}{ cHCO3 (mmol/l) } & G1 & $18,96(2,48)^{\mathrm{AB}, \mathrm{a}}$ & $18,5(2,09)^{\mathrm{A}, \mathrm{a}}$ \\
\hline & G2 & $15,80(4,19)^{\mathrm{B}, \mathrm{a}}$ & $20,38(8,23)^{A, ~ a ~}$ \\
\hline & G3 & $19,30(1,42)^{\mathrm{A}, \mathrm{a}}$ & $19,60(1,69)^{\mathrm{A}, \mathrm{a}}$ \\
\hline \multirow{3}{*}{$\mathrm{ctCO}_{2} \mathrm{~T}(\mathrm{a})$} & G1 & $20,07(2,54)^{\mathrm{AB}, \mathrm{a}}$ & $19,25(2,38)^{\mathrm{A}, \mathrm{a}}$ \\
\hline & G2 & $16,7(4,45)^{\mathrm{B}, \mathrm{a}}$ & $21,62(8,50)^{\mathrm{A}, \mathrm{a}}$ \\
\hline & G3 & $20,4(1,50)^{\mathrm{A}, \mathrm{a}}$ & $20,7(41)^{\mathrm{A}, \mathrm{a}}$ \\
\hline \multirow{3}{*}{ cBase(a) } & G1 & $-5,6(2,66)^{\mathrm{A}, \mathrm{a}}$ & $-5,26(3,03)^{\mathrm{A}, \mathrm{a}}$ \\
\hline & $\mathrm{G} 2$ & $-9,3(4,00)^{\mathrm{B}, \mathrm{a}}$ & $-5(6,69)^{\mathrm{A}, \mathrm{a}}$ \\
\hline & G3 & $-5,5(1,7)^{\mathrm{A}, \mathrm{a}}$ & $-5,4(2,0)^{\mathrm{A}, \mathrm{a}}$ \\
\hline
\end{tabular}

Para cada variável, médias seguidas de letras iguais maiúsculas na coluna não diferem entre si segundo o teste de Tukey e minúsculas na linha não diferem entre si segundo o teste $\mathrm{t}(\mathrm{P}<0,05)$.

\section{CONCLUSÕES}

O remifentanil promoveu incremento da analgesia, permitindo redução de $33 \%$ na velocidade de infusão do propofol. No entanto, a infusão de $0,2 \mu \mathrm{g} / \mathrm{kg} / \mathrm{min}$ de remifentanil determinou maior depressão respiratória quando comparada à infusão de $0,1 \mu \mathrm{g} / \mathrm{kg} / \mathrm{min}$.

\section{COMITÊ DE ÉTICA}

Esta pesquisa foi aprovada pela Comissão de Ética na Experimentação Animal do Departamento de Veterinária da Universidade Federal de Viçosa sob o protocolo número 30/2005, em agosto de 2005.

\section{AGRADECIMENTOS}

À FAPEMIG, pela bolsa de estudos concedida.

\section{REFERÊNCIAS}

Andress JL, Day TK \& Day DG (1995) The effects of consecutive day propofol anesthesia on feline red blood cells. Veterinary Surgery, 24:277-282.

Booth NH \& McDonald LC (1992) Farmacologia e Terapêutica em Veterinária, 6a ed. Rio de Janeiro, Guanabara Koogan. 997p.

Brearley JC, Kellagher REB \& Hall W (1988) Propofol anaesthesia in cats. The Journal of Small Animal Practice, 29:315-322.

Burkle H, Dunbar S \& Van Aken H (1996) Remifentanil: A novel short-acting m-opióide. Anaesthesia \& Analgesia, 83:646-651. 
Correa MA, Aguiar AJ, Neto FJ, Mendes GM, Steagall PV \& Lima AF. (2007) Effects of remifentanil infusion regimens on cardiovascular function and responses to noxious stimulation in propofol-anesthetized cats. American Journal of Veterinary Research, 68:932-40.

Cortopassi SRG \& Fantoni DT (2002) Medicação pré-anestésica. In: Fantoni DT \& Cortopassi SRG (Eds) Anestesia em cães e gatos. Rio de Janeiro, Roca. p.151-158.

Duke T (1995) A new intravenous anesthetic agent: Propofol. Canadian Veterinary Journal, 36:181-183.

Fantoni DT, Cortopassi SRG \& Bernadi MM (1996) Anestésicos intravenosos e outros parenterais. In: Spinosa HS, Górniak SL \& Bernardi MM (Eds) Farmacologia aplicada à medicina veterinária. Rio de Janeiro, Guanabara Koogan. p.104-114.

Glass PSA, Gan TJ \& Howell S (1999) A review of pharmakocinetics and pharmacodinamics of remifentanil. Anesthesia \& Analgesia, 89:7-14.

Hall LH \& Clarke KW (1991) Medicação pré-anestésica. In: Hall LH \& Clarke KW (Eds) Veterinary Anaesthesia. London, Bailliére Tindall. p.50.

Hogue CW Jr, Bowdle TA, O’Leary C, Duncalf D, Miguel R, Pitts M, Streisand J, Kirvassilis G, Jamerson B, McNeal S \& Batenhorst R. (1996) A multicenter evaluation of total intravenous anesthesia with remifentanil and propofol for elective inpatient surgery. Anesthesia and Analgesia, 83:279-85.

James MK, Voung A, Grizzle MK, Schuster SV \& Shaffer JE (1992) Hemodynamic effects of GI87084B, an ultra-short acting $\mathrm{Mu}$ opióide analgesic, in anesthetized dogs. The Journal of Pharmacology and Experimental Therapeutics, 263:84-90.

Jhaveri R, Joshi P, Batenhorst R, Baughman V \& Glass PS (1997) Dose comparison of remifentanil and alfentanil for loss of consciousness. Anesthesiology, 87:253-259.

Langley MS \& Heel RC (1988) Propofol: A review of its pharmacodynamic and pharmacokinetic properties and use as an intravenous anesthetic properties and use as an intravenous anesthetic. Drugs, 35:334-372.

McDonell W (1996) Respiratory system. In: Thurmon JC, Tranquilli WJ \& Benson GJ (Eds) Lumb \& Jones’ Veterinary Anesthesia. Baltimore, Williams \& Wilkins. p.115-147.
Mertens MJ, Engbers FH, Burm AG, Vuyk J (2003) Predictive performance of computer-controlled infusion of remifentanil during propofol/remifentanil anaesthesia. British Journal of Anaesthesia, 90:132-41.

Middleton DJ, Ilkiw JE \& Watson ADJ (1981) Arterial and venous blood gas tension in clinically healthy cats. American Journal of Veterinary Research, 42:1609-1611.

Michelsen LG, Salmenpera M, Hug Jr CC, Szlam F \& VanderMeer D (1996) Anesthetic potency of remifentanil in dogs. Anesthesiology, 84:865-872.

Morgan DWT \& Legge K (1989) Clinical evaluation of propofol as an intravenous anaesthetic agent in cats and dogs. The Veterinary Record, 124:31-33.

Murrel JC, Van Notten RW \& Hellebrekers LJ (2005) Clinical investigation of remifentanil for the total intravenous anaesthesia of dogs. The Veterinary Record, 156:804-808.

Nora FS \& Fortis EAF (2001) Remifentanil: porquê precisamos de outro opióide? Revista Brasileira de Anestesiologia, 51:146-159.

Sano T, Nishimura R, Mochizuki M, Hara Y, Tagawa M \& Sasaki N. (2003) Clinical usefulness of propofol as an anesthetic induction agent in dogs and cats. Journal of Veterinary Medicine Science, 65:641-643.

Short CE \& Bufalari A (1999) Propofol anesthesia. The Veterinary Clinics of North American - Small Animal Practice, 29:747-778.

Souza A P, Pompermayer LG, Lavor MSL, Duarte TS \& Silva RMN (2002) Butorfanol na anestesia com propofol em gatas pré-tratadas com levomepromazina. Ciência Rural, 32:589-594.

Souza A P, Pompermayer LG, Antunes F, Araújo IC \& Silva RMN (2003) Anestesia por infusão contínua e doses fracionadas de propofol em gatos pré-tratados com acepromazina. ARS Veterinária, 19:119-125.

Videira RLR \& Cruz JRS (2004) Remifentanil na prática clínica. Revista Brasileira de Anestesiologia, 54:114-128.

Weaver BMQ \& Raptopoulos D (1990) Induction of anaesthesia in dogs and cats with propofol. Veterinary Record, 126:617-620.

Yasbek KVB (2002) Hipotermia. In: Fantoni DT \& Cortopassi SRG (Eds) Anestesia em cães e gatos. Rio de Janeiro, Roca. p.373-380. 